\title{
Patient-centered care coordination in population health case management
}

\begin{abstract}
While driving through any American city, it is easy to see that neighborhoods differ dramatically. Some are blessed with amenities that promote optimal health and wellbeing for their residents such as parks, playgrounds, walking trails supermarkets that provide healthy food options, fresh fruits and vegetables; buildings in good repair and hospitals within close proximity. All of these amenities/attributes of healthy living are designed to promote social and health success. Healthy people make healthy communities and healthy communities make healthy societies. It must be noted however, that medical health needs are best satisfied and health services are easier to access if you are insured or possess health literacy. Additionally, care is even better if it is coordinated.
\end{abstract}

Patient - centered care coordination by various members of the clinical team will assure enhanced outcomes through self-management support, telehealth, transition of care, engagement of Health Information Technology, managing acute and chronic conditions, ongoing communication, facilitating coordination among health care providers and the health delivery system. Case Managers who are charged with the assignment to coordinate all aspects of the care of their patients are able to complete this assignment most successfully when working with members of the interdisciplinary team and successfully crossing many disciplines. Case Management led by Nursing Case Managers has been identified as a key driver in value-based healthcare delivery as well as a cornerstone of the Patient Protection and Affordable Care Act enacted in 2010 under the Obama Administration. Purposeful case management interventions across the health continuum are resulting in more efficient and appropriate care coordination leading to improved patient outcomes, increased satisfaction with care delivery and increased compliance with the Centers for Medicare and Medicaid Services (CMS) regulations by hospitals across the nation and closing the gap in health disparities. Case Managers and their leadership in Care Coordination continues to be most successful clinically, socially and financially in addressing the determinants of health and meeting the overall needs of patients and their families from a population health perspective.
Volume 5 Issue 4 - 2018

\author{
Alleson M Sailsman, Jamesetta A Halley- \\ Boyce, Ann M Sailsman \\ Health Systems Administration Programs, College of Nursing \\ Seton Hall University, USA
}

Correspondence: Jamesetta A Halley-Boyce, Health Systems Administration Programs, College of Nursing Seton Hall University, USA, Tel+ 347-742-0138, Email halleyja@shu.edu

Received: June 30, 2018 | Published: August 08, 2018

\section{Determinants of health}

As consumers of healthcare we experience varying levels of care delivery and even treatment modalities which may vary by socioeconomic stratification even amongst those that have health insurance. Documented research in Cardiovascular Medicine has shown that treatment may differ by race, ethnicity, and by the zip code in which one live, work and play. ${ }^{1}$ Studies have also revealed significant mortality resulting from social conditions such as low education, racial segregation, low social support, income inequality and individual/area poverty. ${ }^{2}$

Health care is fragmented at best and in addition to the aforementioned, stakeholders and interests groups, legislators, policymakers and politics, have significant impact on healthcare for both individuals and populations. The chasm between patients and outcomes is also built into reimbursement structures. An overarching reform that is poised to reduce the fragmented system of health care is the Patient Protection and Affordable Care Act of 2010 (ACA). This health reform act will extend insurance coverage to as many as 32 million Americans. The law also included regulations that affect the quality of coverage insurers must offer. Additionally, the law created a range of initiatives focused on encouraging reform in how medical care is organized and delivered, with a goal of reducing costs and improving quality outcomes. Finally, other aspects of the law provided funding for expanded primary care capacity and a wide range of other health systems improvements. ${ }^{3}$

Value - based purchasing has added another dimension as the Centers for Medicare and Medicaid Services have added linkages between costs and quality through value based purchasing and bundled payments. The linkages between quality, quantity cost, are being tested by health care organizations. The aging population with concomitant increases in chronic illnesses and enhanced use of technology, including medical informatics, have also driven the cost of healthcare higher. ${ }^{4}$ Through better management of case it is anticipated that we can improve the quality of care, Improve overall efficiency and reduce health disparities.

Case Management is not a new concept but, has become a "hot topic" with the advent of the ACA. ${ }^{5}$ Case Management makes a difference in the quality of the care patients receive and supports the theory that case managers can improve consumer satisfaction. The Institute of Medicine (IOM) clearly support this initiative of patient centered care and the need for improvement in care delivery and further feels that case management can control these factors including cost containment. This is based on the evidence that patients undergoing complex health problems, catastrophic health and social situations need assistance to navigate the healthcare systems ${ }^{6}$ we concur. 
The ultimate goal of case management is to simultaneously promote the patient's wellness, autonomy and appropriate use of service and financial resources. In the Case Management Society of America (CMSA) Standards of Practice for Case Management, case management is defined as a "collaborative process of assessment, planning, facilitation, care coordination, evaluation and advocacy for options and services to facilitate an individual's and family's comprehensive health needs through communication and available resources to promote quality and cost effective outcomes (CMSA, 2016)." The focus will be on the care coordination aspect of case management.

\section{Care coordination}

The National Quality Forum (NQF) defined care coordination as "process that is personalized, safe, high quality care that is provided to the patient and family across the health care continuum and human service. It therefore includes effective integration of the services and personnel from different care settings, and disciplines." (NQF, 2010) incorporates the use of health information technology systems (HIT) for information transfer and sharing as instant accessibility to information will ensure the delivery of better health care.

\section{The five key domains of care coordination are-}

\section{Health home or patient centered medical home (PCMH)}

This can be a community medical center, a physician, or a clinic. This model of care strives to be patient-focused; coordinated and accessible, not fragmented as is the case for numerous medically disadvantage communities. According to Taliani et al., ${ }^{7}$ for care management to be an effective component of the PCMH, care managers need to be available during the patient encounter to meet with them. There needs to be support for patient self-management, utilization of Electronic Medical Record (EMR) for team messaging and patient tracking, as well as ongoing communication and a commitment to organizing and coordinating care that reflects the patient's needs, preferences, priorities, and values. The patient is treated as a unique individual, and his/her point of view is incorporated in the decision making process, thus, driving improved outcomes, reduction in unnecessary hospital readmissions, coordination of unfragmented care and improved quality and safety of care. The underlying concept of patient -centered care is to treat the patient as a person rather than as a disease or illness. It is having a compassionate presence, providing for the physical and emotional needs of the patient. Constructing a culture of patient centered care requires the clinician to be knowledgeable of clinical practices.

To achieve patient-centered care at the organizational level, the following factors must be considered:

\section{Leadership/vision/supportive environment}

A unique leadership style and an embracing, supportive environment are considered most important of all factors whether or not the care setting is in a hospital, ambulatory, community, medical home or personal home. Support must be given and leaders should be committed to promoting this care process. The priorities and processes should support the leader's vision of the organization. The vision's strategic elements should be articulated on a daily basis, while simultaneously fostering a supportive environment for the workforce to grow and thrive. Provide organizational members with the same respect and dignity that is afforded to patients with the goal of employee loyalty and commitment to the success of the leader's vision.

\section{Systemic measurement and feedback}

In order to gain success in patient- centered care, organizations have to listen and systematically monitor performance based on feedback from service users. Healthcare should not just be for the patients but for the providers of service. Patients and families should also be engaged in their health and wellbeing and be able to make informed decisions about their care. Health literacy must be promoted to assure patients and their families are knowledgeable, informed, active participants in the plan of care. The patients must first be educated in order to make the right decisions. Additionally, supportive technology such as Health Information Technology (HIT) encourages patients and families to gain access to their personal health information and to communicate with their care provider. Economic constraints and the aim to provide better outcomes are drivers of patient - centered care.

\section{A proactive plan of care and follow up}

A written Plan of Care should anticipate the patient's needs and also provide a tracking system of the patient's progress towards goal achievement. The evolution of care plans result in various terms and methods namely critical paths, critical pathways, care maps, multidisciplinary action plans, nursing care plans. Regardless of the title, standards of clinical guidelines using evidence based practices must be presented. It must include patient's medications, and follow up appointments, serving as a handoff between clinicians, between care settings, and is particularly helpful with patients with multi morbidity, poly pharmacy status and those who are usually older with some degree of impairment in mobility and Activities of Daily Living. Many patients experience challenges of self-management when living with multiple chronic conditions. The importance of supporting patient's self-management is key to improving health status and selfefficacy. Symptoms of multi morbidity are inter dependent, so the plan of care should address all the issues and potential issues. ${ }^{8}$

\section{Communication}

Care coordination is a collaborative process, therefore communication is a hallmark for success and is critical across all care settings and sectors, particularly during care transitions. Effective communication can reduce unnecessary re-admissions. Systematically assessing for understanding and communication throughout the care continuum is an essential component of comprehensive health care, particularly for lower income adult population and medically complex patients. There is wide spread availability and use of HIT including electronic health records and Regional Health Information Organizations (RHIO). Health information exchange tools are available to facilitate real time data sharing and will improve care coordination. ${ }^{9}$

\section{Health information technology (HIT)}

The emergence of HIT has made a difference in our health care system, though challenges still remain. Several studies strongly indicate advancement in several informatics areas within patientcentered care that have been impactful in care coordination. Namely, team care, care transitions, clinical decision support, personal health records, and telehealth, all of which support patient-centered care. ${ }^{10}$ 
Of particular importance is its potential to help achieve the population health Triple Aim: Improved patient care, improved population health and reduction in healthcare costs. HIT comprises of various components, hardware, workflows, e-prescriptions, computerized physicians order entry, clinical decisions support, electronic health records, and patient engagement tools. ${ }^{3}$

Health Information Exchange (HIE) allows the physicians in a network to access and retrieve information about patients across providers and settings. A necessary component of HIT is interoperability the ability of different systems to communicate with .each other. The goal is to have information available to teams of physicians, nurses and care coordinators in a private, secure way. When information is shared in a timely fashion it can improve diagnoses, reduce duplication of testing, prevent readmissions and avoid medication errors.

According to Pollack et al., ${ }^{11}$ HIT is improving patient care. A study assessed the care of patients with end stage renal disease in three dialysis units. It found that patient mortality increased when nursing staffing was determined to be inadequate and decreased upon the implementation of EHRs (Electronic Health Records) Another study ${ }^{12}$ conducted at 41 hospitals found that hospitals with more advanced HIT, experienced fewer complications, lower mortality and lower health care costs than those with less sophisticated electronic systems.

HIT has the potential to make health care more uniformed from one setting to another and overtime can lead to more standardized care. Lowering the costs of health care is a component of the Triple Aim. The current cost of health care is $18 \%$ of Gross domestic product. The adoption of HIT has grown especially due to the growing importance of the following models of integrated care - Affordable Care Organizations (ACOs), Patient Centered Medical Home (PCMH) and Health Homes that are growing as the health reform becomes more advanced and all three are structured to contain the exorbitant health care costs

In the PCMH model of care, HIT links all the caregivers electronically. If a patient sees a Behavioral Health Specialist for depression but, does not discuss his emotional wellbeing with his other providers, they will be able to see this information. The ACOs is a contract between a payer and a group of providers. The ACO assigns a group of patients to the providers and hold the provider responsible for the quality of care provided, the patient's experience and the total costs of care. ${ }^{6}$

A health home has goals similar to PCMH and targets the 5\% of the population that account for $50 \%$ of all health care costs. These patients have multiple issues, social and medical and non-medical issues are addressed in the total care approach. All parties have vested interest in obtaining patient information. Other technology such as virtual care, smart phone, computers are all making healthcare information more accessible.

\section{Transitions of care}

Transitional Care is a continuous process in which a patient's care shifts from being provided in one setting of care to another such as from a hospital to home, a skilled nursing facility or even to another hospital. The Institute of Medicine (IOM) report Crossing the Quality Chasm describes the United States health system as decentralized, complicated and poorly organized, specifically noting the "layers of processes and handoffs that patients and families find bewildering and clinicians view as wasteful" The IOM noted that patients receive inadequate information when leaving one setting to another on the aftercare - information, on medication usage and sides effects, activities, how to care for (IOM, 2001). Follow up appointments are sometimes not done or are unclear or inadequate and as a result many patients are readmitted to hospitals within 30 days of discharge. ${ }^{13}$

There are many factors at play when there is a poor transition of patient care, for example the differences in computer systems, primary care physicians are not aware that patients have been admitted or discharged and some patients will be lost to follow up. Several models of care transition improvement have been developed and tested. A model that is widely used is the Care Transitions Intervention developed by Eric Coleman at the University of Colorado - Patients are provided with transition coaches to promote the development of patient's skills in areas such as medication management, scheduling and follow up care, responding to alerts to notify physician or other healthcare provider, seek emergency services and take full ownership of their health.

Another plan developed by Mary Naylor and her colleagues (2011) at the University of Pennsylvania involved a longer period of intervention and an Advanced Practice RN who coaches patients and their care giver to better manage their care and pursue follow up plans with Primary Care providers. Over $14 \%$ of all patients hospitalized in the united states are readmitted within 30 days of discharge, patient of low socioeconomic status are $43 \%$ more likely to be readmitted. Numerous studies have been conducted using administrative data, but few studies focused on patients perception of readmission and lack of understanding of the patient's perspective are some reasons that interventions may not always be as successful for patients in the transition process. ${ }^{14}$

Effective care transition management is the key to achieving value-based care for patients. Transitions should ideally be patient centered and should not disrupt the continuum process, however, delays, interruptions and unnecessary costs and ultimately frustration for patients and physicians are hurdles that need to be overcome. The authors further state that there are five keys to managing transitions of care.

i. Formalize your inbound patient access by managing the patient through follow up communication

ii. Focus on logistics. There has to be an understanding of how information flows, address patient care handoff and provide a telephone number or secure web based access for transition team members

iii. Ensure reimbursement is obtained

iv. Determine new ways to coordinate-better communication, educate patient about care plan, instructions on follow up and appropriate contacts during transitions and finally,

v. Focus on preventing readmission to hospital. Attention should be paid to high risks patients, such as patients with Heart failure exacerbation, chronic obstructive pulmonary disease and history of an active smoker, patients with uncontrolled diabetes, etc. Ensure fragmentations in care are minimized. Refer patient to a home care agency and for tele health management for chronic disease management. ${ }^{15-19}$

\section{Conclusion}

Patient- centered care coordination in population health case management approach provides the "building blocks" in addressing the determinants of population health. The model supports the promotion 
of successful population health delivery which promotes the Triple Aim: lower cost, better care, and better patient experience. It facilitates access to quality care, supports clinical decision making, provides risk reduction strategies, supports patient engagement in the coordination of care across the life course and the care continuum, thus measuring and improving outcomes. Patient-centered care coordination provides the arena for the various care models to interplay and focus on chronic and preventative care management, and/or complex patient needs. The goals of improving health outcomes, reducing hospital readmissions and costs, improving safety and quality care, and promoting successful care transitions must be actualized in care coordination. In addressing the determinants of population health through patientcentered care coordination; particularly in patients with continuous complex medical care that require more frequent care in multiple care settings, it is incumbent on healthcare practitioners to be well trained and be competent with the various concepts in caring for patients with complex acute and chronic illnesses. To reduce morbidity and mortality and assure the best overall patient outcomes, those clinicians who are most therapeutically effective will possess current knowledge as well as be appraised of the patient's goals, preferences and clinical status.

Patient -centered care coordination can be for an individual or multi-level patient population health approach. The common themes are patients with increased complexity, social vulnerability, multiple care providers and varying abilities for self-care. Groups that may benefit from care coordination are patients with complex medical and social needs, special populations such as the elderly, pediatric patients with special needs, behavioral health patients, disabled, multiple chronic illness, and patients with cognitive impairment. Ultimately, care coordination process has broad applicability in multiple settings of care from the primary care clinic to the specialty single practice to more complex systems such as ACOs. Simply put, Care Coordination improves outcomes for both the individual and the population while simultaneously enhancing the satisfaction of the caregiver and the clinical members of the Inter-professional Care Team with regard to their delivery of care that is safe, therapeutic, cost effective and compassionate.

\section{Acknowledgments}

None.

\section{Conflict of interest}

The author declares that there is no conflict of interest.

\section{References}

1. Agarwal SE, Tuzcu and Kapadia. Choice and Selection of Treatment Modalities for Cardiac Patients: An Interventional Cardiology Perspective. J Am Heart Assoc. 2015;4(10):e002353.

2. Galea S, Tracy M, Hoggatt KJ, et al. Estimated deaths attributable to social factors in the United States. Am J Public Health. 2011;101(8):1456-1465
3. Knickman J, Kovner A. Health Care Delivery in the United States. $11^{\text {th }}$ edition. New York, NY: Springer Publishing Company; 2015.

4. Cesta T, Hussein M. The Case Manager's Survival Guide. Winning Strategies in the New Health Care Environment. $3^{\text {rd }}$ edition. Lancaster PA: DEStech Publication; 2016.

5. Amaded K. 2010 Patient Protection and Affordability Care Act Summary. 2017.

6. Finkelman AW. Case Management for Nurses. Upper Saddle River: NJ Prentice Hall; 2011.

7. Taliani CA, Bricker PL, Adelman AM, et al. Implementing effective care management in the patient-centered medical home. Am J Manag Care. 2013;19(12):957-964.

8. Stanhope M, Lancaster J. Public Health Nursing. Population Centered health Care in The Community. $8^{\text {th }}$ edition. Maryland Heights: Elsevier; 2014.

9. Cipriano P, Bowles K, Daley M, et al. The Importance of Health Information Technology in Care Coordination and Transitional Care. Nursing Outlook. 2013;61(6):475-489.

10. Demiris G, Kneale, L. Informatics Systems and Tools to Facilitate Patient-centered Care Coordination. Yearbook of Medical Informatics, 2015;10(1):15-21.

11. Pollack V, Lorch J. Effect of electronic patient record use on mortality in end Stage renal disease, a model chronic disease: Retrospective analysis of 9 years of retrospectively collected data. BMC Med Inform Decis Mak. $2007 ; 7: 38$.

12. Amarasingham R, Plantinga L, Diener West M, et al. Clinical Information Technologies and Inpatient outcomes. Arch Intern Med. 2009;169(2):108-114.

13. Case Management Society of America. Standards of Practice for Case Management, 2016.

14. Coleman E. Falling through the Cracks: Challenges and Opportunities for Improving Transitional Care for Persons with Continuous Complex Care Needs. J Am Geriatr Soc. 2003;51(4):549-555.

15. Coleman E, Parry C, Chalmers S, et al. The Care Transitions interventions, results of Randomized Controlled Trial. Arch Intern Med. 2009;166(17):1822-1887.

16. Halley-Boyce J, McLaughlin. Case Management in Transitional Care. JOCEPS. 2014;58(1).

17. Healthcare Intelligence Network. Case Management Metrics: Charting Care Coordination across the Healthcare Continuum. 2011.

18. National Quality Forum. Quality Connections: Care Coordination, 2010

19. Institute of Medicine. Crossing the Quality Chasm: A New Health Systemfor the 21st Century. Vol. 6. Washington DC: National Academy Press; 2001 